\author{
VALERIA FRANCARDI $(*)$ - ANNARITA CITO $(*)$ - SARA FUSI $(* *)$ - MAURIZIO BOTTA $(* * *)$ \\ ELENA DREASSI $(* *)$
}

\title{
LINSEED TO INCREASE N-3 FATTY ACIDS IN TENEBRIO MOLITOR (COLEOPTERA TENEBRIONIDAE)
}

\author{
(*) Consiglio per la ricerca in agricoltura e analisi dell'economia agraria - Research Centre for Plant Protection and \\ Certification, Via Lanciola 12/A, 50125 Firenze, Italy. \\ (**) Department of Biotechnology, Chemistry and Pharmacy, University of Siena, via A. Moro, 53100 Siena, Italy \\ (***) Lead Discovery Siena Srl, via Vittorio Alfieri 31, 53019 Castelnuovo Berardenga - Siena, Italy \\ Corresponding authors: Dreassi E., e-mail: elena.dreassi@unisi.it; Francardi V., e-mail: valeria.francardi@crea.gov.it
}

Francardi V., Cito A., Fusi S., Botta M., Dreassi E. - Linseed to increase n-3 fatty acids in Tenebrio molitor (Coleoptera Tenebrionidae).

The yellow mealworm Tenebrio molitor is one of the most promising edible insect species for human consumption and its potential use as a supplement of unsaturated fatty acids (UFA) for human diet, has been recently confirmed

The possibility to enhance polyunsaturated acids (PUFA), especially omega-3 (n-3) content, was evaluated in $T$. molitor mealworms. The purpose was to obtain an n-6/n-3 ratio more suitable for human consumption, which is useful for the secondary prevention of cardiovascular diseases. To this end, tests were carried out by adding linseed, as source of n-3 acid, to insect feeding diets. A decrease of saturated fatty acids (SFA) and an increase of PUFA contents, especially n-3 acid, was simultaneously observed in larvae fed on almost all the diets enriched with linseed. As a result, supplementation of feeding diets with linseed determined a favorable insect growth rate and a decrease of n-6/n-3 ratio to values more suitable for the prevention and treatment of cardiovascular diseases.

KEY WoRDS: Tenebrio molitor; n-6/n-3 ratio; polyunsaturated fatty acids (PUFA); $\alpha$-linolenic acid; linseed.

\section{INTRODUCTION}

The potential use of the yellow mealworm T. molitor larvae as unsaturated fatty acids (UFA) supplement for human diet has been recently proposed (DREASSI et al., 2017). The fat component of this coleopteran is indeed relatively low in saturated fatty acids (SFA) content but high in unsaturated fatty acids (UFA) content, mainly oleic acid (18:1n-9), among the monounsaturated fatty acids (MUFA); $\alpha$-linoleic acid (18:2n-6) (n-6) and $\alpha$-linolenic acid (18:3n3) (n-3) among the polyunsaturated fatty acids (PUFA). LiVINGSTONE et al. (2012) reported that a high content of UFA in human diet might have beneficial effects on the cardiovascular system, partially replacing SFA intake.

PUFA, especially eicosapentaenoic acid (20:5n-3, EPA) and docosahexaenoic acid (22:6n-3, DHA), the long chain derivatives of $\alpha$-linolenic acid, are the main fatty acids (FA) involved in the prevention of cardiovascular diseases (MANERBA et al., 2010). The mealworm T. molitor is able to synthesize de novo $\alpha$-linoleic and $\alpha$-linolenic acid; conversely, it does not contain EPA and DHA, as similarly observed in other terrestrial insect species (DADD, 1983; SÁNCHEZ-Muros et al., 2014).

Though a lower $n-6 / n-3$ ratio (4:1) is reported as the most favourable value for the secondary cardiovascular prevention (Simopoulos, 2002), the mealworm T. molitor is generally characterized by a $n-6 / n-3$ ratio suitable for human consumption, ranging between 20:1 to 25:1 (DREASSI et al., 2017).

Diets are known to alter insect growth rate and nutritional quality and this suggests the possibility to increase the production and nutritional composition of insects to better suit consumer needs (ANDERSON, 2000; RICCIARDI \& BAVIERA, 2016). In fact, several studies confirmed the possibility to increase PUFA content in insects through diet; for example, diets supplemented with fish offal increased n3 fatty acid in the dipteran Hermetia illucens (L.) (Diptera: Stratiomyidae) (ST-HILAIRE et al., 2007).

Insect nutritional quality may change also according to the insect developmental stage, as observed in a recent study that reported a lower $\mathrm{n}-6 / \mathrm{n}-3$ ratio in $T$. molitor larvae than in pupae raised on diets with a growing fat content (DREASSI et al., 2017).

The aim of the present study is to enhance PUFA content, especially n-3 FA, in T. molitor larvae to obtain n-6/n-3 ratios more similar to values recommended for human secondary prevention of cardiovascular diseases. For this purpose T. molitor larvae were reared on different diets suitable for T. molitor feeding (DREASSI et al. 2017), and supplemented with commercial linseed, known as "health seed" for its nutritive properties. Fat composition of larvae reared on the enriched diets was compared to those previously fed on the corresponding base diets.

The influence of the enriched diets on T. molitor larval growth was also investigated.

\section{MATERIALS AND METHODS}

Four different base diets (D3-D6), previously selected for T. molitor larvae mass rearing (DREASSI et al., 2017), were supplemented with linseed ground to flour $(10 \% \mathrm{w} / \mathrm{w})$ and used as feeding substrates (hereafter indicated as D3+-D6+). FA compositions of the diets were determined on the basis of a single analysis. Carrot pieces were added to each diet twice a week to provide the tenebrionid with a moisture source.

T. molitor larvae were obtained from the commercial 
supplier "La Voliera" (Florence, Italy) but detailed information on the diet they had been previously provided with was not available. In the laboratory, larvae were divided in four groups and separately reared on the base diets (D3-D6) to complete their development until the adult stage. 250 young larvae (15-20 mm length) of the following generation were collected from each diet and reared on the corresponding diet enriched with linseed (D3+-D6+).

Fat content and fatty acids (FA) composition analysis was carried out from the insects reared on the enriched diets (D3+$\mathrm{D} 6+)$ in the same conditions previously reported for the base diets (D3-D6) (DrEASsi et al., 2017). The influence of the enriched diets on T. molitor mealworms growth rate, based on the number of days elapsed from the beginning of the tests until or beyond 50\% pupation (hereafter referred to as PT50) and cumulative mortality percentage (hereafter referred to as mortality) were evaluated, as previously described (DREASSI et al., 2017). Finally, fifty $T$. molitor newly formed pupae (free from chitin exuvia and excreta) were weighed and the obtained medium values used as an indirect index of the best feeding diet for the tenebrionid fitness, as performed for other coleopteran species (HunT et al., 1992).
FA analysis of lipid fraction in all the diets and insects were performed with GC-FID and GC-MS, as previously reported (DREASSI et al., 2017).

Quantitative analyses (fat content and FA composition) were run at least in triplicate and data expressed as means \pm standard deviations (SD). A T-test was performed to assess significant differences in fat content and FA composition between larvae reared on each base diet and those reared on the same diet enriched with linseed. Differences in growth parameters (PT50, cumulative mortality percentage and pupal medium weight) were also evaluated. All data were tested for normality and transformations (log and angular transformation respectively) were then applied before statistical analysis.

\section{RESULTS AND DISCUSSION}

FAT CONTENT AND FA COMPOSITION OF THE BREEDING DIETS

The diets enriched with linseed (D3+-D6+) showed an increase of $3-3.5 \%$ in total fat content based on dry matter compared to the base diets (D3-D6) (Table 1).

Table 1 - Fat content and FA composition of the rearing diets used for Tenebrio molitor larvae.

\begin{tabular}{|c|c|c|c|c|c|c|c|c|}
\hline & \multicolumn{8}{|c|}{ Diets $^{\mathrm{a}}$} \\
\hline & $\mathrm{D} 3^{\mathrm{b}}$ & D3+ & $\mathrm{D} 4^{\mathrm{b}}$ & D4+ & $\mathrm{D}^{\mathrm{b}}$ & D5+ & $\mathrm{D}^{\mathrm{b}}$ & D6+ \\
\hline $\begin{array}{l}\text { Total fat content } \\
\text { (\% dry weight) }\end{array}$ & 6.23 & 9.81 & 7.34 & 10.81 & 7.92 & 11.33 & 9.34 & 12.61 \\
\hline \multicolumn{9}{|l|}{$\mathrm{FA}(\mathrm{mol} \%)$} \\
\hline Capric acid 10:0 & 0.02 & 0.02 & 0.00 & 0.03 & 0.23 & 0.14 & 0.26 & 0.10 \\
\hline Lauric acid 12:0 & 0.14 & 0.00 & 0.06 & 0.03 & 0.12 & 0.08 & 0.22 & 0.07 \\
\hline Tridecylic acid 13:0 & 0.00 & 0.00 & 0.00 & 0.00 & 0.00 & 0.00 & 0.00 & 0.00 \\
\hline Myristic acid 14:0 & 0.39 & 0.23 & 0.40 & 0.36 & 0.58 & 0.45 & 0.34 & 0.28 \\
\hline $14: 1 n-5$ acid & 0.00 & 0.00 & 0.00 & 0.00 & 0.00 & 0.00 & 0.00 & 0.00 \\
\hline $14: 2 n-3$ acid & 0.00 & 0.00 & 0.00 & 0.00 & 0.00 & 0.00 & 0.12 & 0.00 \\
\hline Myristoleic acid $14: 1 n-5$ & 0.00 & 0.00 & 0.00 & 0.00 & 0.00 & 0.00 & 0.00 & 0.00 \\
\hline Pentadecylic acid 15:0 & 0.08 & 0.06 & 0.03 & 0.03 & 0.03 & 0.05 & 0.00 & 0.05 \\
\hline Palmitic acid 16:0 & 16.18 & 5.78 & 19.55 & 15.36 & 19.40 & 16.04 & 19.31 & 13.19 \\
\hline $16: 1 n-5$ acid & 0.26 & 0.04 & 0.26 & 0.07 & 0.68 & 0.07 & 0.70 & 0.04 \\
\hline Palmitoleic acid 16:1n-7 & 0.16 & 0.17 & 0.20 & 0.28 & 0.17 & 0.69 & 0.00 & 0.67 \\
\hline $16: 2 n-4$ & 0.00 & 0.00 & 0.00 & 0.00 & 0.00 & 0.00 & 0.00 & 0.00 \\
\hline Margaric acid 17:0 & 0.06 & 0.09 & 0.03 & 0.09 & 0.04 & 0.09 & 0.12 & 0.07 \\
\hline $17: 1 n-6$ acid & 0.00 & 0.03 & 0.00 & 0.03 & 0.00 & 0.02 & 0.00 & 0.03 \\
\hline Stearic acid 18:0 & 2.12 & 3.94 & 1.84 & 2.76 & 1.96 & 2.94 & 1.98 & 3.59 \\
\hline Oleic acid $18: 1 n-9$ & 31.55 & 24.62 & 35.84 & 30.61 & 35.72 & 28.07 & 34.68 & 24.14 \\
\hline$\alpha$-Linoleic acid $18: 2 n-6$ & 46.07 & 33.62 & 39.64 & 32.25 & 38.93 & 30.50 & 40.28 & 24.61 \\
\hline Arachidic acid 20:0 & 0.43 & 0.33 & 0.15 & 0.14 & 0.14 & 0.14 & 0.14 & 0.13 \\
\hline$\alpha$-Linolenic acid $18: 3 n-3$ & 1.81 & 30.94 & 1.34 & 17.65 & 1.35 & 20.31 & 1.37 & 32.49 \\
\hline Eicosenoic acid 20:1n-9 & 0.46 & 0.11 & 0.54 & 0.27 & 0.51 & 0.34 & 0.48 & 0.55 \\
\hline $20: 2 n-6$ acid & 0.05 & 0.03 & 0.05 & 0.04 & 0.04 & 0.07 & 0.00 & 0.00 \\
\hline Behenic acid 22:0 & 0.21 & 0.00 & 0.07 & 0.00 & 0.07 & 0.00 & 0.00 & 0.00 \\
\hline SFA & 19.63 & 10.45 & 22.13 & 18.81 & 22.59 & 19.94 & 22.37 & 17.48 \\
\hline MUFA & 32.44 & 24.96 & 36.83 & 31.26 & 37.08 & 29.18 & 35.86 & 25.42 \\
\hline PUFA & 47.93 & 64.59 & 41.04 & 49.93 & 40.33 & 50.88 & 41.78 & 57.10 \\
\hline SFA/UFA & 0.24 & 0.12 & 0.28 & 0.23 & 0.29 & 0.25 & 0.29 & 0.21 \\
\hline$n-6 / n-3$ ratio & 25.51 & 1.09 & 29.54 & 1.83 & 28.79 & 1.51 & 26.91 & 0.76 \\
\hline
\end{tabular}

${ }^{a}$ Diets composition (\%w/w): D3 wheat flour (25), oat flour (25), corn flour (25), chickpea flour (25); D3+ linseed (10), wheat flour (22.5), oat flour (22.5), corn flour (22.5), chickpea flour (22.5); D4 oat flour (50), wheat flour (50); D4+ linseed (10), oat flour (45), wheat flour (45); D5 beer yeast (5), wheat flour (47.5), oat flour (47.5); D5+ linseed (10), beer yeast (5), wheat flour (42.5), oat flour (42.5); D6 beer yeast (0.5), wheat flour (33.17), oat flour (33.17), corn flour (33.17); D6+ linseed (10), beer yeast (0.5), wheat flour (29.8), oat flour (29.8), corn flour (29.8);

${ }^{\mathrm{b}}$ data previously reported by DREASSI et al. (2017). 
Though sharing the same most prevalent FA, the four enriched diets showed a lower SFA content, prevalently palmitic acid, while stearic acid slightly increased. Moreover, the MUFA content, mainly oleic acid, decreased compared with that recorded in base diets. In addition, a higher PUFA content was detected in all the diets enriched with linseed $v s$ the corresponding base diets; $\alpha$-linolenic acid content was 20-30 times higher in enriched $v s$ base diets; conversely, $\alpha$-linoleic content decreased after supplementation of each diet with linseed. As a result, the higher amount of $\alpha$-linolenic acid determined a decrease of $n-6 / n-3$ ratio in all the enriched diets (Table 1 ).

FAT CONTENT AND FA COMPOSITION OF THE MEALWORMS

The increased fat amount detected in the diets enriched with linseed was not observed in the mealworms fed on them. In fact, the mealworms fed on the enriched diets showed a total fat content similar to that of insects fed on the corresponding base diets (Table 2). The same most prevalent FA (myristic acid, palmitic acid, 16:1n-5 acid, palmitoleic acid, stearic acid, oleic acid, $\alpha$-linoleic and $\alpha$-linolenic acid) were found either in $T$. molitor larvae fed on the base diets or in those fed on the corresponding enriched diets. Conversely, a significantly decrease of SFA, prevalently palmitic acid, was detected in specimens fed on each enriched diet $v s$ the corresponding base diet. A significantly decrease of MUFA, mainly oleic acid, was also detected in larvae fed on D3+, D4+ and D6+ diets, balanced by a significantly higher PUFA content that entailed a significant decrease of SFA/UFA ratio. Finally, the addition of the linseed $(10 \% \mathrm{w} / \mathrm{w})$ led to an increase of $\alpha$-linolenic acid content in larvae and, consequently, to a significant decrease of the n6/n-3 ratio in the mealworms fed on enriched diets (Table 2).

\section{T. MOLITOR LARVAE GROWTH AND DEVELOPMENTAL RATE}

Growth rate data of $T$. molitor are reported in Table 3 . The mealworms reared on D3+ diet reached or exceed $50 \%$ pupation (PT50) in significantly fewer days than those fed

Table 2 -Fat content and FA composition of Tenebrio molitor larvae fed on four different diets and the same diets enriched with linseed $(10 \% \mathrm{w} / \mathrm{w})$.

\begin{tabular}{|c|c|c|c|c|c|c|c|c|}
\hline & \multicolumn{8}{|c|}{ Diets } \\
\hline & $\mathrm{D}^{\mathrm{a}}$ & D3+ & $\mathrm{D} 4^{\mathrm{a}}$ & D4+ & D5 $^{\mathrm{a}}$ & D5+ & D6 $^{\mathrm{a}}$ & D6+ \\
\hline $\begin{array}{l}\text { Total fat content } \\
\text { (\% dry weight) }\end{array}$ & $16.76 \pm 1.32$ & $15.88 \pm 0.69$ & $15.48 \pm 1.86$ & $16.01 \pm 1.45$ & $15.61 \pm 2.43$ & $16.84 \pm 1.55$ & $15.58 \pm 0.87$ & $14.88 \pm 1.24$ \\
\hline \multicolumn{9}{|l|}{ Fatty acid (mol\%) } \\
\hline Capric acid 10:0 & $0.03 \pm 0.00$ & $0.06 \pm 0.01$ & $0.05 \pm 0.01$ & $0.05 \pm 0.00$ & $0.03 \pm 0.00$ & $0.03 \pm 0.01$ & $0.03 \pm 0.00$ & $0.05 \pm 0.00 *$ \\
\hline Lauric acid 12:0 & $0.72 \pm 0.06$ & $0.45 \pm 0.04^{*}$ & $0.79 \pm 0.06$ & $0.44 \pm 0.07^{*}$ & $0.78 \pm 0.09$ & $0.65 \pm 0.02$ & $0.78 \pm 0.11$ & $0.65 \pm 0.06$ \\
\hline Tridecylic acid 13:0 & $0.06 \pm 0.01$ & $0.04 \pm 0.01 *$ & $0.09 \pm 0.14$ & $0.04 \pm 0.01$ & $0.10 \pm 0.02$ & $0.14 \pm 0.02$ & $0.09 \pm 0.01$ & $0.13 \pm 0.06^{*}$ \\
\hline Myristic acid 14:0 & $6.79 \pm 1.44$ & $4.95 \pm 0.28$ & $6.99 \pm 0.86$ & $4.64 \pm 0.37$ & $6.98 \pm 1.12$ & $6.80 \pm 0.62$ & $7.21 \pm 1.30$ & $6.63 \pm 0.44$ \\
\hline $14: \ln -5$ acid & $0.40 \pm 0.10$ & $0.30 \pm 0.01$ & $0.43 \pm 0.07$ & $0.27 \pm 0.00$ & $0.50 \pm 0.14$ & $0.38 \pm 0.01$ & $0.45 \pm 0.11$ & $0.33 \pm 0.03$ \\
\hline $14: 2 n-3$ acid & $0.19 \pm 0.03$ & $0.17 \pm 0.01$ & $0.21 \pm 0.02$ & $0.15 \pm 0.03$ & $0.25 \pm 0.06$ & $0.40 \pm 0.09$ & $0.22 \pm 0.03$ & $0.30 \pm 0.05$ \\
\hline Myristoleic acid 14:1n-5 & $0.01 \pm 0.00$ & $0.01 \pm 0.01$ & $0.01 \pm 0.00$ & $0.01 \pm 0.00$ & $0.01 \pm 0.00$ & $0.02 \pm 0.00$ & $0.01 \pm 0.00$ & $0.04 \pm 0.00^{* *}$ \\
\hline Pentadecylic acid 15:0 & $0.08 \pm 0.00$ & $0.07 \pm 0.02$ & $0.09 \pm 0.01$ & $0.08 \pm 0.00$ & $0.09 \pm 0.00$ & $0.10 \pm 0.01$ & $0.08 \pm 0.01$ & $0.09 \pm 0.01$ \\
\hline Palmitic acid 16:0 & $20.79 \pm 0.79$ & $17.11 \pm 1.33^{*}$ & $19.46 \pm 0.37$ & $16.63 \pm 0.72 * *$ & $20.06 \pm 1.14$ & $15.71 \pm 0.40^{*}$ & $20.52 \pm 0.55$ & $15.95 \pm 0.52^{* *}$ \\
\hline $16: 1 n-5$ acid & $1.37 \pm 0.33$ & $0.78 \pm 0.07$ & $1.46 \pm 0.18$ & $0.61 \pm 0.01 *$ & $1.60 \pm 0.34$ & $1.32 \pm 0.03$ & $1.66 \pm 0.30$ & $1.20 \pm 0.08$ \\
\hline Palmitoleic acid 16:1n-7 & $1.82 \pm 0.15$ & $2.59 \pm 0.12 *$ & $1.74 \pm 0.11$ & $2.51 \pm 0.15^{* *}$ & $2.10 \pm 0.21$ & $3.15 \pm 0.39^{*}$ & $1.94 \pm 0.07$ & $2.85 \pm 0.11^{* *}$ \\
\hline Margaric acid 17:0 & $0.06 \pm 0.02$ & $0.09 \pm 0.01$ & $0.07 \pm 0.01$ & $0.11 \pm 0.02 *$ & $0.07 \pm 0.01$ & $0.09 \pm 0.00^{*}$ & $0.06 \pm 0.01$ & $0.07 \pm 0.03$ \\
\hline $16: 2 n-4$ acid & $0.26 \pm 0.02$ & $0.18 \pm 0.03 *$ & $0.27 \pm 0.01$ & $0.17 \pm 0.01 * *$ & $0.32 \pm 0.08$ & $0.15 \pm 0.03$ & $0.24 \pm 0.02$ & $0.24 \pm 0.02$ \\
\hline $\mathrm{ni}\left(\mathrm{t}_{\mathrm{R}} 34.1 \mathrm{~min}\right)$ & $0.05 \pm 0.01$ & $0.39 \pm 0.28$ & $0.07 \pm 0.02$ & $0.31 \pm 0.14$ & $0.06 \pm 0.02$ & $0.12 \pm 0.12$ & $0.04 \pm 0.01$ & $0.25 \pm 0.05^{* *}$ \\
\hline $17: 1 \mathrm{n}-6$ acid & $0.05 \pm 0.01$ & $0.07 \pm 0.01$ & $0.05 \pm 0.01$ & $0.08 \pm 0.00^{*}$ & $0.06 \pm 0.01$ & $0.27 \pm 0.25$ & $0.05 \pm 0.01$ & $0.09 \pm 0.01 *$ \\
\hline Stearic acid 18:0 & $3.36 \pm 0.56$ & $3.67 \pm 0.58$ & $3.31 \pm 0.06$ & $3.96 \pm 0.81$ & $2.82 \pm 0.24$ & $2.68 \pm 0.79$ & $3.20 \pm 0.13$ & $3.12 \pm 0.25$ \\
\hline Oleic acid $18: 1 n-9$ & $44.80 \pm 0.46$ & $42.78 \pm 1.11^{*}$ & $44.53 \pm 1.28$ & $40.89 \pm 0.97 *$ & $43.60 \pm 2.07$ & $43.81 \pm 1.13$ & $46.21 \pm 0.57$ & $42.91 \pm 1.47 * *$ \\
\hline $\mathrm{ni}\left(\mathrm{t}_{\mathrm{R}} 39.5 \mathrm{~min}\right)$ & $0.17 \pm 0.01$ & $0.19 \pm 0.00^{*}$ & $0.18 \pm 0.00$ & $0.22 \pm 0.00^{* *}$ & $0.18 \pm 0.01$ & $0.26 \pm 0.05$ & $0.18 \pm 0.00$ & $0.24 \pm 0.02 *$ \\
\hline$\alpha$-Linoleic acid $18: 2 \mathrm{n}-6$ & $18.51 \pm 1.51$ & $22.32 \pm 1.69$ & $19.71 \pm 0.87$ & $23.16 \pm 1.70$ & $19.84 \pm 2.47$ & $19.29 \pm 1.09$ & $16.63 \pm 1.41$ & $20.65 \pm 0.16^{* *}$ \\
\hline Arachidic acid 20:0 & $0.10 \pm 0.03$ & $0.15 \pm 0.04$ & $0.10 \pm 0.02$ & $0.14 \pm 0.04$ & $0.09 \pm 0.03$ & $0.11 \pm 0.01$ & $0.10 \pm 0.03$ & $0.10 \pm 0.00$ \\
\hline$\alpha$-Linolenic acid $18: 3 n-3$ & $0.28 \pm 0.12$ & $3.35 \pm 0.11 * *$ & $0.28 \pm 0.08$ & $5.25 \pm 0.35^{* *}$ & $0.32 \pm 0.09$ & $4.18 \pm 0.62 * *$ & $0.23 \pm 0.09$ & $4.83 \pm 0.21^{* *}$ \\
\hline Eicosenoic acid 20:1n-9 & $0.03 \pm 0.04$ & $0.05 \pm 0.00$ & $0.04 \pm 0.01$ & $0.06 \pm 0.02$ & $0.05 \pm 0.02$ & $0.06 \pm 0.01$ & $0.04 \pm 0.02$ & $0.06 \pm 0.01$ \\
\hline $20: 2 n-6$ acid & $0.02 \pm 0.01$ & $0.12 \pm 0.05^{*}$ & $0.02 \pm 0.01$ & $0.11 \pm 0.01 * *$ & $0.02 \pm 0.01$ & $0.18 \pm 0.02 * *$ & $0.02 \pm 0.01$ & $0.11 \pm 0.01 * *$ \\
\hline $\mathrm{ni}\left(\mathrm{t}_{\mathrm{R}} 47.8 \mathrm{~min}\right)$ & $0.03 \pm 0.00$ & $0.11 \pm 0.04$ & $0.06 \pm 0.02$ & $0.12 \pm 0.02 *$ & $0.05 \pm 0.01$ & $0.07 \pm 0.04$ & $0.02 \pm 0.02$ & $0.10 \pm 0.01 *$ \\
\hline SFA & $31.99 \pm 0.84$ & $26.58 \pm 1.08^{* *}$ & $30.94 \pm 0.66$ & $26.08 \pm 0.34 * *$ & $31.03 \pm 0.80$ & $26.31 \pm 0.25^{* *}$ & $32.06 \pm 0.94$ & $25.80 \pm 1.28^{* *}$ \\
\hline MUFA & $48.49 \pm 0.84$ & $46.77 \pm 1.06^{*}$ & $48.26 \pm 1.03$ & $44.61 \pm 1.17 *$ & $47.92 \pm 1.85$ & $49.18 \pm 1.31$ & $50.37 \pm 0.76$ & $47.71 \pm 1.24^{*}$ \\
\hline PUFA & $19.26 \pm 1.64$ & $25.96 \pm 1.82 *$ & $20.48 \pm 0.90$ & $28.66 \pm 1.32 * *$ & $20.75 \pm 2.64$ & $24.06 \pm 1.60$ & $17.33 \pm 1.51$ & $25.90 \pm 0.00^{* *}$ \\
\hline SFA/UFA & $0.47 \pm 0.02$ & $0.37 \pm 0.02 * *$ & $0.45 \pm 0.01$ & $0.36 \pm 0.01 * *$ & $0.45 \pm 0.02$ & $0.36 \pm 0.01 * *$ & $0.47 \pm 0.02$ & $0.35 \pm 0.02 * *$ \\
\hline n-6/n-3 ratio & $39.06 \pm 2.89$ & $6.38 \pm 0.28^{* *}$ & $40.53 \pm 0.85$ & $4.33 \pm 0.59^{* *}$ & $34.68 \pm 3.09$ & $4.29 \pm 0.16^{* *}$ & $37.05 \pm 3.05$ & $4.05 \pm 0.12 *$ \\
\hline
\end{tabular}

a data previously reported by DREASSI et al. (2017).

$* * *$ Values significantly different between mealworms fed on each base diet $v s$ the corresponding enriched $\operatorname{diet}(* \mathrm{p}<0.05 ; * * \mathrm{p}<0.01)$ $\mathrm{ni}=$ not identified 
Table 3 - Tenebrio molitor mean days $( \pm \mathrm{SD})$ to reach PT50, cumulative mean percentage mortality and pupal medium weight $(\mathrm{n}=50$ pupae) fed on the different diets.

\begin{tabular}{|c|c|c|c|c|c|c|c|c|}
\hline & $\mathrm{D} 3^{\mathrm{a}}$ & D3+ & $\mathrm{D} 4^{\mathrm{a}}$ & D4+ & $\mathrm{D}^{\mathrm{a}}$ & D5+ & D6 $^{\mathrm{a}}$ & D6+ \\
\hline $\begin{array}{l}\text { Days to reach } \\
\text { PT50 (days) }\end{array}$ & $188.00 \pm 17.35^{*}$ & $159.60 \pm 9.61 *$ & $100.40 \pm 17.49$ & $126.40 \pm 7.76^{* *}$ & $96.20 \pm 3.83$ & $88.40 \pm 20.84$ & $111.00 \pm 23.01$ & $83.00 \pm 12.30$ \\
\hline $\begin{array}{l}\text { Cumulative } \\
\text { mortality (\%) }\end{array}$ & $46.80 \pm 3.63$ & $39.20 \pm 6.57$ & $20.80 \pm 13.39$ & $13.20 \pm 11.37$ & $30.40 \pm 10.62$ & $39.60 \pm 7.67$ & $29.60 \pm 11.87$ & $38.00 \pm 15.62$ \\
\hline $\begin{array}{l}\text { Pupal weight } \\
\text { (mg) }\end{array}$ & $109.18 \pm 16.62$ & $115.84 \pm 20.07 * *$ & $132.68 \pm 15.84$ & $120.80 \pm 17.70$ & $144.38 \pm 16.61$ & $156.42 \pm 25.38 * *$ & $138.96 \pm 22.05$ & $164.46 \pm 15.74 * *$ \\
\hline
\end{tabular}

a data previously reported in DREASSI et al. (2017).

$* * *$ Values significantly different between mealworms fed on each base diet $v s$ the corresponding enriched diet $(* \mathrm{p}<0.05 ; * * \mathrm{p}<0.01)$

on D3, while PT50 of $T$ molitor reared on D4+ was recorded in significantly more days than that recorded in insects reared on D4. The shortest PT50s were observed for the mealworms fed on D5+ (88.40 days) and D6+ diets (83.00 days) even if results were not significantly different from those fed on the corresponding base diets. Moreover, no significant differences were highlighted between cumulative mortality percentages of the mealworms fed on the base diets (D4-D6) and those fed on the same diets enriched with linseed (D4+-D6+).

Pupae fed on D3+, D5+ and D6+ diets were significantly heavier than those fed on the corresponding base diets.

The enrichment of the feeding diet of the mealworm $T$. molitor with linseed opens a concrete possibility of tenebrionid employment in preparation of human food supplements with interesting functional properties for human health: low SFA intake and an optimal n-6/n-3 ratio for cardiovascular prevention (Simopoulos, 2002). Furthermore, the enrichment of the feeding diets with linseed produced at the same time a high quality of the fat fraction and a favourable insect growth rate.

\section{ACKNOWLEDGMENTS}

Research carried out under the project "The insects and prevention cardiovascular diseases - INSECT CARD" funded by the Tuscany Region - Public Call for Research Projects in the Nutraceutical industry. Executive Decree no. 4741 of 21 October 2014; n. 5418 of 11.17.2014. The Authors thank Dr. Francesco Binazzi for the linguistic review of the paper.

\section{REFERENCES}

ANDERSON S.J., 2000 - Increasing calcium levels in cultured insects. - Zoo. Biol., 19: 1-9.
Dreassi E., Cito A., Zanfini A., Materozzi L., Botta M., FRANCARDI V., 2017 - Dietary fatty acids influence the growth and fatty acid composition of the yellow mealworm Tenebrio molitor (Coleoptera: Tenebrionidae). - Lipids, 52 (3): 285-294; doi:10.1007/s11745-016-4220-3.

DADD R.H., 1983 - Essential fatty acids: insects and vertebrates compared. In Metabolic Aspects of Lipid Nutrition in Insects. - T.E. Mittler, R.H. Dadd, eds., Boulder, Colo.: Westview Press pp. 107-147.

Hunt D.W.A., DruRY C.F., MAW H.E., 1992 - Influence of nitrogen in the performance of Colorado Potato Beetle (Coleoptera: Chrysomelidae) on tomato. - Environ. Entomol., 21: 817-821.

Livingstone K.M., Lovegrove J.A., Givens D.I., 2012 The impact of substituting SFA in dairy products with MUFA or PUFA on CVD risk: evidence from human intervention studies. - Nutr. Res. Rev., 25: 193-206.

Manerba A., Vizzardi E., Metra M., Dei CAS L., 2010 n-3 PUFAs and cardiovascular disease prevention. Future Cardiol, 6: 343-350.

RiCCIARDI C., BAVIERA C., 2016 - Role of carbohydrates and proteins in maximizing productivity in Alphitobius diaperinus (Coleoptera Tenebrionidae). - Redia, 99: $97-$ 105; http://dx.doi.org/10.19263/REDIA-99.16.13.

SÁnchez-Muros M.J., Barroso F.G., Manzano-AguGLIARO F., 2014 - Insect meal as renewable source of food for animal feeding: a review. - J. Cleaner Prod., 65: 16-27.

Simopoulos A.P., 2002 - The importance of the ratio of omega-6/omega-3 essential fatty acids. - Biomed Pharmacother, 56: 365-379.

St-Hilaire S., Cranfill K., McGuire M.A., Mosley E.E., Tomberlin J.K., Newton L., Irving S., 2007 - Fish Offal Recycling by the Black Soldier Fly Produces a Foodstuff High in omega - 3 Fatty Acids. - J. World Aquacult. Soc., 38: 309-313. 